\title{
Periodic Solutions in the Planar $(n+1)$ Ring Problem with Oblateness
}

\author{
Antonio Elipe* $*$ and Mercedes Arribas $\ddagger$ \\ Universidad de Zaragoza, 50009 Zaragoza, Spain \\ and \\ Tilemahos J. Kalvouridis \\ National Technical University of Athens, 15773 Athens, Greece
}

DOI: $\underline{10.2514 / 1.29524}$

\begin{abstract}
In the $N$-body ring problem, the motion of an infinitesimal particle attracted by the gravitational field of $(n+1)$ bodies is studied. These bodies are arranged in a planar ring configuration. This configuration consists of $n$ primaries of equal mass $m$ located at the vertices of a regular polygon that is rotating on its own plane about its center of mass with a constant angular velocity $w$. Another primary of mass $m_{0}=\beta m(\beta \geq 0$ parameter $)$ is placed at the center of the ring. Moreover, we assume that the central body may be an ellipsoid, or a radiation source, which introduces a new parameter $\epsilon$. In this case, the dynamics are found to be much richer than the classical problem due to the different equilibria and bifurcation characteristics. We find families of periodic orbits and make an analysis of the orbits by studying their evolution and stability along the family for several values of the new parameter introduced.
\end{abstract}

\section{Introduction}

$\mathbf{L}$ ET us consider $n+1$ bodies, $P_{0}, P_{1}, \ldots, P_{n}$, such that points $P_{1}, \ldots, P_{n}$ have identical masses $m$, and the mass of $P_{0}$ is $m_{0}=\beta m$, with $\beta \geq 0$ a parameter. The points are arranged in such a way that points $P_{1}, \ldots, P_{n}$ are at the vertices of the regular $n$ gon, whereas $P_{0}$ is at the center of this $n$ gon. This configuration is known in the literature $[1,2]$ as the $N$-ring configuration.

This kind of configuration is considered as a model of observed phenomena such as planetary rings, some stellar formations, asteroids, the motion of an artificial satellite about a ring, etc.

The problem may be considered a classic one. For instance, Maxwell [3] in 1856 studied the stability of a discrete particle ring and Tisserand [4] in 1889 reformulated Maxwell's analysis and presented a relation between the mass of each ring particle and the number of them in order that the system be linearly stable. However, the problem has recently attracted the attention mainly due to the rich dynamics of the motion of an infinitesimal particle under the attraction of the gravitational field of this multibody configuration presents.

Scheeres [2] investigated this configuration including Hill stability, invariant transformations, equilibrium points, periodic orbits, etc. Kalvouridis $[1,5,6]$ studied the stationary solutions and the zero-velocity curves and surfaces, and found symmetric periodic motions for the particular cases $n=4,5$. In previous work by two of the authors [7], the evolution with $\beta$ of the equilibrium points was analyzed and the value of the mass parameter $\beta$ for which bifurcations exist was obtained. Furthermore, the problem was extended to consider a central body that is nonspherical or a radiation source (see [8]) and so, a new parameter $\epsilon$ is introduced. With this modification, we obtained the equilibria and values of the bifurcations in the parametric plane $\beta-\epsilon$, which shows big changes in the topology of the extended problem with respect to the classical one.

Presented as Paper 6748 at the AIAA/AAS Astrodynamics Specialist, Keystone, Colorado, 21-24 August 2006; received 29 December 2006; revision received 27 June 2007; accepted for publication 29 June 2007. Copyright $\odot 2007$ by the American Institute of Aeronautics and Astronautics, Inc. All rights reserved. Copies of this paper may be made for personal or internal use, on condition that the copier pay the $\$ 10.00$ per-copy fee to the Copyright Clearance Center, Inc., 222 Rosewood Drive, Danvers, MA 01923; include the code 0731-5090/07 \$10.00 in correspondence with the CCC.

*Professor, Grupo de Mecánica Espacial-IUMA. Associate Fellow AIAA.

${ }^{\dagger}$ Associate Professor, Grupo de Mecánica Espacial.

Frofessor, Department of Mechanics.
The introduction of this new parameter $\epsilon$ models several problems, for instance, where the central body of the ring is no longer spherical, but an ellipsoid of revolution (spheroid). In natural bodies like planets, the spheroid is oblate $(\epsilon>0)$, but we can also think of artificial bodies and assume them to be prolate, in that case, $\epsilon<0$. We can also think of the central body to be a radiations source, and then, the effect of the radiation can be modeled in a similar way to the oblate ellipsoid (see, for instance, [8] ). We can also think of adding a relativistic correction to the potential of the main body, which gives rise to Manev's type potential $([9,10])$. For the above cases and for quasi-homogeneous potentials [10-12], it is proven in [13] that, as it happens with Newtonian forces, the configuration is homographic; that is, it remains self-similar along the time.

Periodic orbits give most of the information available in complex dynamical systems. Recently, Pinotsis [14] obtained families of periodic orbits for this problem with Newtonian forces. We extend this work here by finding families of periodic orbits for several values of $\epsilon<0$ and fixed values $n=7$ and $\beta=2.0$. We considered negative values of the parameter because, as shown in [7], the dynamics are more complex for negatives values. We focus our attention in $x$ symmetric orbits, and although we present here representative families with bifurcations in mind, the analysis has been quite exhaustive.

\section{Equations of Motion}

To describe the motion of the infinitesimal particle let us take a planar reference frame rotating at an angular velocity $w$, such that the origin is at the center of the $n$ gon, and the $x$ axis is on the line joining the central body with one of the primaries on the ring, say $P_{1}$; the remaining bodies are numbered counterclockwise.

With a suitable choice of units of time and length, the equations of motion in this synodic frame (Fig. 1) can be expressed as (see [7] for details)

$$
\ddot{x}-2 \dot{y}=-\frac{\partial U}{\partial x}, \quad \ddot{y}+2 \dot{x}=-\frac{\partial U}{\partial y}
$$

where the effective potential is

$$
U(x, y)=-\frac{1}{2}\left(x^{2}+y^{2}\right)-\frac{1}{\Delta}\left[\beta\left(\frac{1}{r_{0}}+\frac{\epsilon}{r_{0}^{2}}\right)+\sum_{i=1}^{n} \frac{1}{r_{i}}\right]
$$

$r_{0}$ is the distance of the particle to the central body and $r_{i}$ the distance to the mass $m_{i}$, given by 


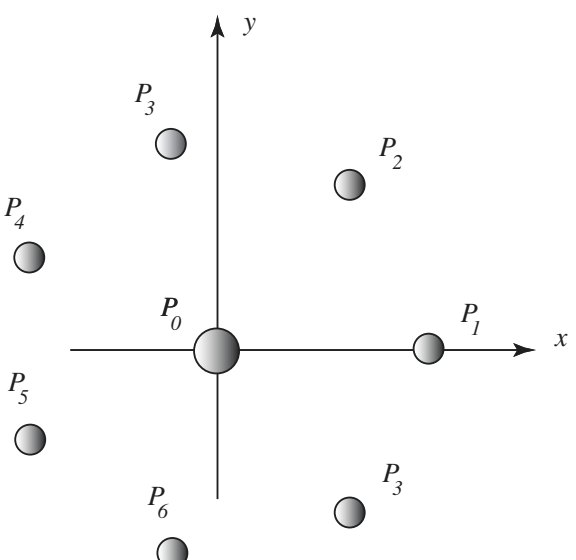

Fig. 1 The $n+1$ ring configuration in the synodic frame.

$$
r_{0}=\sqrt{x^{2}+y^{2}}, \quad r_{i}=\sqrt{\left(x-x_{i}\right)^{2}+\left(y-y_{i}\right)^{2}}, \quad i=1, \ldots, n
$$

By introducing the angle $\theta=\pi / n$, the coordinates $\left(x_{i}, y_{i}\right)$ of the peripheral $i$ th body are

$$
x_{i}=\frac{1}{M} \cos 2(i-1) \theta, \quad y_{i}=\frac{1}{M} \sin 2(i-1) \theta
$$

with $M=2 \sin \theta$, and $\Delta=M\left(\Lambda+\beta M^{2}\right)$ is a constant depending on the parameter $\beta$ and the number $n$ of primaries through

$$
\Lambda=\sin ^{2} \theta \sum_{i=2}^{n} \frac{1}{\sin (i-1) \theta}
$$

The parameter $\epsilon$, due to the nonsphericity of the central primary, can be either positive or negative.

Note that system (1) has the Jacobian first integral

$$
C=2 U+\left(\dot{x}^{2}+\dot{y}^{2}\right)
$$

As is well known, equilibria are obtained by solving the algebraic system

$$
\frac{\partial U}{\partial x}=0, \quad \frac{\partial U}{\partial y}=0
$$

and their stability depends on the roots of the characteristic equation:

$$
\lambda^{4}+\left(4+U_{x x}+U_{y y}\right) \lambda^{2}+\left(U_{x x} U_{y y}-U_{x y}^{2}\right)=0
$$

As shown in [7], the most interesting situation is obtained when the parameter $\epsilon$ is negative. Figure 2 shows that the number of equilibria changes depending on $\epsilon$. Equilibria (see [7]) are either on the $x$ axis or on the bisector between two primaries (and their equivalent by symmetries), and, because we are dealing with an odd number of primaries, by cutting the potential with the plane $y=0$, equilibria will lie on this line, as we see in Fig. 2 for several values of $\epsilon$. Bifurcation lines in parametric plane $\beta-\epsilon$ were obtained in [7].

\section{Families of Periodic Orbits}

For the numerical determination of periodic orbits, we fixed the value of the parameter $\beta=2$ and take three different values of $\epsilon$, namely, $\epsilon=-0.1,-0.14$, and -0.3 ; the reason for this choice is that for the first and last values the number of equilibria are different, and the intermediate value is close to the bifurcation value (see Fig. 2).

We focus our attention on periodic orbits that are symmetric with respect to the $x$ axis; that is, they perpendicularly cross the $x$ axis exactly twice per period. They are known as simple periodic orbits. These symmetric orbits exist because of the symmetries of the problem. Indeed, the transformation
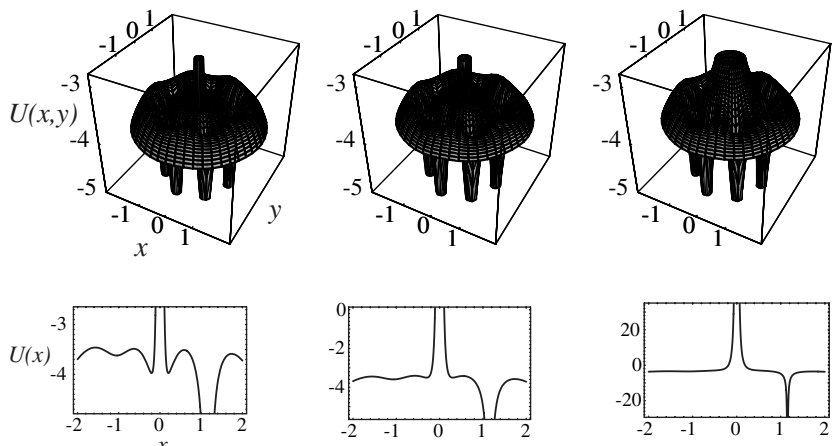

Fig. 2 The potential function $U(x, y)$ (top row) and the section $U(x)$ $(y=0)$ (bottom row) for $n=7, \beta=2$, and $\epsilon=-0.1$ (left), $\epsilon=-0.14$ (center), and $\epsilon=-0.3$ (right). For $\epsilon=-0.1$ and $\epsilon=-0.14$ there are seven equilibria, whereas for $\epsilon=-0.3$ there is only one equilibrium.

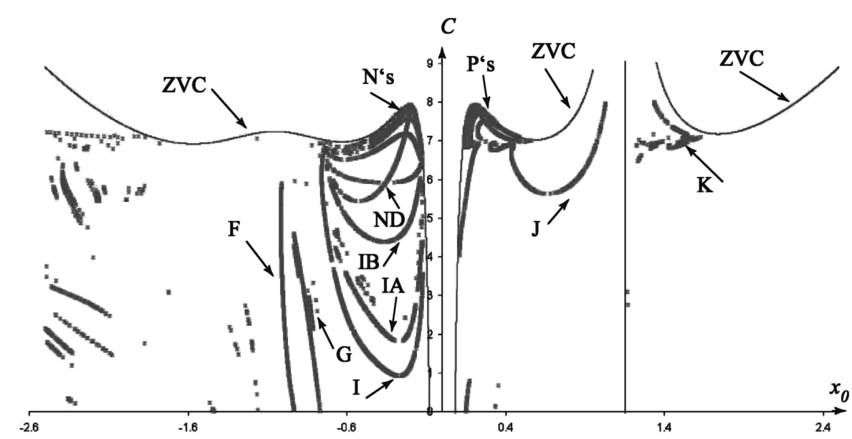

Fig. 3 Families of periodic orbits for $\epsilon=-0.1$ on the plane $x_{0}-C$.

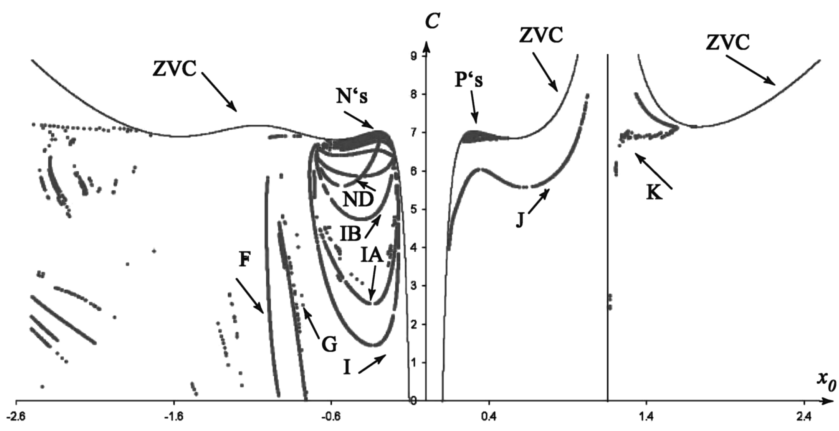

Fig. 4 Families of periodic orbits for $\epsilon=-0.14$ on the plane $x_{0}-C$.

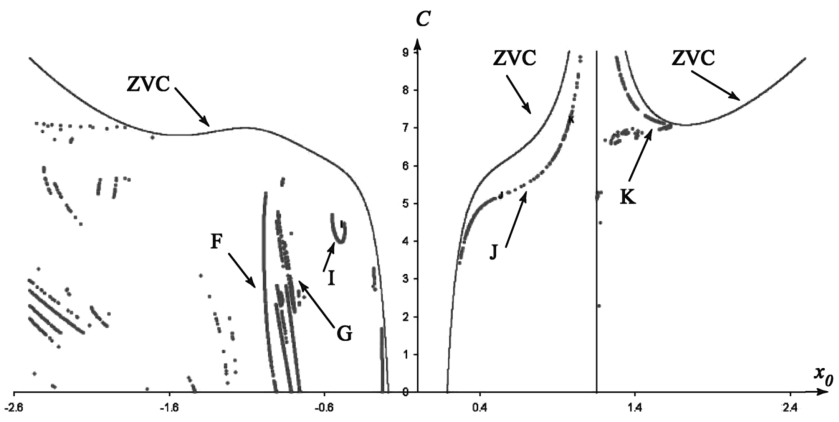

Fig. 5 Families of periodic orbits for $\epsilon=-0.3$ on the plane $x_{0}-C$. 


$$
(x, y, \dot{x}, \dot{y}, t) \rightarrow(x,-y,-\dot{x}, \dot{y},-t)
$$

leaves equations of motion (1) invariant.

Thus, the initial conditions (at $t=0$ ) for a such symmetric periodic orbit are

$$
\left(x_{0}, y_{0}, \dot{x}_{0}, \dot{y}_{0}\right)=\left(x_{0}, 0,0, \dot{y}_{0}\right)
$$

whereas at instant $T / 2$ (half-period), the solution must be

$$
\left(x_{T / 2}, y_{T / 2}, \dot{x}_{T / 2}, \dot{y}_{T / 2}\right)=\left(x_{T / 2}, 0,0, \dot{y}_{T / 2}\right)
$$

Among the existing methods of finding periodic orbits we have selected the grid-search method [15], because for our case it is quite efficient. Let us outline the procedure used. Solutions of the system (1) are continuous functions of the initial conditions and time and, besides, this system has the Jacobian constant (3), hence for given values of the Jacobian constant $C$ and the initial condition $x_{0}$, we calculate $\dot{y}_{0}$. Then, we keep the value of $C$ and make a small variation of $x_{0}$ by increasing it in $\delta x_{0}$. For each value of $x_{0}$ we numerically integrate the equations of motion until the orbit crosses again the axis of symmetry (simple periodic orbits). Let $T / 2$ be this time. For each orbit, we check the sign of $\dot{x}(T / 2)$. If for two successive orbits corresponding to $x_{0}$ and $x_{0}^{\prime}=x_{0}+\delta x_{0}$, the velocity $\dot{x}(T / 2)$ changes its sign, that is, if $\dot{x}(T / 2) \dot{x}^{\prime}(T / 2)<0$, then, because of the continuity of the solutions, there exists a value $x_{0}^{*} \in\left[x_{0}, x_{0}^{\prime}\right]$ for which $\dot{x}(T / 2)=0$, which means a symmetric periodic solution. We record every interval $\left[x_{0}, x_{0}^{\prime}\right]$ where a change of sign of $\dot{x}(T / 2)$ occurs. The same procedure is repeated with another value of $C^{\prime}$ slightly different from the previous one $\left(C^{\prime}=C+\delta C\right)$. Therefore, by double scanning (grid search) the $x_{0}-C$ diagram, we finally build a file containing the intervals where symmetric orbits probably exist. During the scanning process we considered $\dot{y}_{0}>0$. Then, a bisection method is applied to obtain the "exact" initial conditions of the symmetric periodic orbits. We used a Runge-Kutta (7)8 method for the numerical integration and the accuracy in the bisection method was up to $10^{-9}$.

In this paper we name direct orbits as those that are described in the same sense as the rotation of the synodic coordinate system and retrograde orbits as those that are described in the opposite sense. There are cases where some orbits present loops; however, although the sense of the particle motion may reverse along the loops, the dominant character (direct or retrograde) of the trajectory after one period does not change.

The isoenergetic exploration reduces the system to a symplectic map of the plane $(x, \dot{x})$ and the Jacobian matrix of the map is often represented as

$$
H=\left(\begin{array}{ll}
a & b \\
c & d
\end{array}\right)=\left(\begin{array}{ll}
\partial x / \partial x_{0} & \partial x / \partial \dot{x}_{0} \\
\partial \dot{x} / \partial x_{0} & \partial \dot{x} / \partial \dot{x}_{0}
\end{array}\right)
$$

The eigenvalues of this matrix play a major role in discussing the stability of the orbits [16-18]. Indeed, the trace of this matrix $k=a+d$ is called stability index. It was shown by Hénon [16] that

\begin{tabular}{|c|c|c|c|c|}
\hline $\bar{\epsilon}$ & $x_{0}$ & $C$ & $T$ & $\bar{a}$ \\
\hline \multicolumn{5}{|c|}{ Family $F$} \\
\hline \multirow[t]{3}{*}{-0.10} & -1.0110855331 & 5.8599499164 & 8.8832307616 & $0.7659499110 \mathrm{E}+03$ \\
\hline & -0.9892149200 & 1.9999099513 & 5.3947479804 & $0.4381617123 \mathrm{E}+03$ \\
\hline & -0.9309984131 & 0.0299099782 & 4.0539905626 & $0.9532580040 \mathrm{E}+02$ \\
\hline \multirow[t]{3}{*}{-0.14} & -1.0011043091 & 5.8099499377 & 8.8313173300 & $0.7927519924 \mathrm{E}+03$ \\
\hline & -0.9858304291 & 1.9799099537 & 5.3828725760 & $0.4195742383 E+03$ \\
\hline & -0.9328661957 & 0.1499099741 & 4.1375670204 & $0.1016818001 \mathrm{E}+03$ \\
\hline \multirow[t]{3}{*}{-0.30} & -0.9902571564 & 4.0199599381 & 6.8670373872 & $0.8634201683 \mathrm{E}+03$ \\
\hline & -0.9116564178 & 0.0599599762 & 4.0523353818 & $0.8215559381 \mathrm{E}+02$ \\
\hline & -0.7028617024 & -3.9400401718 & 2.1904159986 & $0.1246417341 \mathrm{E}+04$ \\
\hline \multicolumn{5}{|c|}{ Family $G$} \\
\hline \multirow[t]{3}{*}{-0.10} & -0.9349858761 & 4.6099199708 & 4.8199726026 & $0.1147806123 \mathrm{E}+05$ \\
\hline & -0.8518515339 & 2.5899099151 & 2.8188158994 & $0.1465644858 \mathrm{E}+04$ \\
\hline & -0.7686068420 & 0.0099127512 & 2.0067516852 & $0.1277586282 E+04$ \\
\hline \multirow[t]{3}{*}{-0.14} & -0.9299095420 & 4.6499199735 & 4.9253557284 & $0.1161503086 \mathrm{E}+05$ \\
\hline & -0.8527541389 & 2.7899099306 & 2.9270001004 & $0.1406316294 \mathrm{E}+04$ \\
\hline & -0.7619066162 & 0.0299135882 & 2.0048881846 & $0.1075606728 \mathrm{E}+04$ \\
\hline \multirow[t]{3}{*}{-0.30} & -0.8987493801 & 4.7199599787 & 5.2133919420 & $0.8300418807 \mathrm{E}+04$ \\
\hline & -0.8416219826 & 3.3199599519 & 3.2940242666 & $0.8156446723 \mathrm{E}+03$ \\
\hline & -0.7987826996 & 2.2599598512 & 2.6540182094 & $0.2384805346 \mathrm{E}+03$ \\
\hline \multicolumn{5}{|c|}{ Family J } \\
\hline \multirow[t]{3}{*}{-0.10} & 0.1585152552 & 5.7899799902 & 4.1684792882 & $0.5331645772 \mathrm{E}+03$ \\
\hline & 0.4340683326 & 6.6940199983 & 7.3343683016 & $0.1246719776 \mathrm{E}+05$ \\
\hline & 0.6451821777 & 5.6299999945 & 2.6529243726 & $0.2871520686 \mathrm{E}+01$ \\
\hline \multirow[t]{3}{*}{-0.14} & 0.1942219006 & 5.0199799792 & 2.4500238358 & $0.2493613368 \mathrm{E}+02$ \\
\hline & 0.5467590820 & 5.6199799966 & 3.1463868574 & $0.1594904633 \mathrm{E}+02$ \\
\hline & 0.8222134766 & 5.8999799861 & 1.3950738318 & $0.2358273111 \mathrm{E}+00$ \\
\hline \multirow[t]{3}{*}{-0.30} & 0.3604400635 & 4.6199397962 & 1.5157965668 & $0.8049359118 \mathrm{E}+01$ \\
\hline & 0.6709270020 & 5.4399599832 & 1.6365097144 & $0.4884962785 E+00$ \\
\hline & 1.0021380308 & 7.5599597582 & 0.4222308458 & $0.8989564663 \mathrm{E}+00$ \\
\hline \multicolumn{5}{|c|}{ Family $K$} \\
\hline \multirow[t]{3}{*}{-0.10} & 1.5123955750 & 7.0619899980 & 5.5336846026 & $0.2534987037 \mathrm{E}+03$ \\
\hline & 1.5467378907 & 7.1259899963 & 3.8777222810 & $0.1018750378 \mathrm{E}+01$ \\
\hline & 1.3324592285 & 7.9999999569 & 0.7802236906 & $0.7598692333 \mathrm{E}+00$ \\
\hline \multirow[t]{3}{*}{-0.14} & 1.5947729937 & 7.0979899524 & 4.3177613052 & $0.7948886489 E+01$ \\
\hline & 1.5761220212 & 7.1499899841 & 3.3094437092 & $0.1173536816 \mathrm{E}+01$ \\
\hline & 1.3365469971 & 7.9899999499 & 0.7597743728 & $0.7644889895 E+00$ \\
\hline \multirow[t]{3}{*}{-0.30} & 1.6041901466 & 7.0209997906 & 4.5496219946 & $0.2073748542 \mathrm{E}+02$ \\
\hline & 1.6330651705 & 7.0679899474 & 3.9153384204 & $0.3603599386 \mathrm{E}+01$ \\
\hline & 1.3342912598 & 8.0799599230 & 0.6250588960 & $0.8173260030 \mathrm{E}+00$ \\
\hline
\end{tabular}
the stability boundaries are $k=+2$ (two eigenvalues of +1 ) and $k=-2$ (with two eigenvalues of -1 ). The condition of symmetry

Table 1 Initial conditions for orbits from families $F, G, J$, and $K$ and several values of $\epsilon$ 
Table 2 Initial conditions for orbits of families I, IA, IB, N, and NB and several values of $\epsilon$

\begin{tabular}{|c|c|c|c|c|}
\hline$\epsilon$ & $x_{0}$ & $C$ & $T$ & $a$ \\
\hline \multicolumn{5}{|c|}{ Family I } \\
\hline \multirow[t]{3}{*}{-0.10} & -0.1232320557 & 3.4799099802 & 4.4919467454 & $0.6203020298 \mathrm{E}+03$ \\
\hline & -0.1711210175 & 1.5299099621 & 2.6715726408 & $0.5781207512 \mathrm{E}+02$ \\
\hline & -0.5593553910 & 2.0800199821 & 3.6405598702 & $0.8577123565 \mathrm{E}+02$ \\
\hline \multirow[t]{3}{*}{-0.14} & -0.1746691551 & 3.4999099837 & 4.5946836250 & $0.4751288865 E+03$ \\
\hline & -0.2287325195 & 1.8680099845 & 3.0499855936 & $0.3831425480 \mathrm{E}+02$ \\
\hline & -0.6661792141 & 3.6839899818 & 5.0531373302 & $0.8895923628 \mathrm{E}+03$ \\
\hline \multirow[t]{3}{*}{-0.30} & -0.4704693848 & 4.4259999931 & 5.9032806470 & $0.2677450770 \mathrm{E}+03$ \\
\hline & -0.4989142578 & 3.9720299939 & 5.3766171522 & $0.2516848236 \mathrm{E}+02$ \\
\hline & -0.5487824554 & 4.6199599893 & 6.1522975854 & $0.3421697307 \mathrm{E}+03$ \\
\hline \multicolumn{5}{|c|}{ Family IA } \\
\hline \multirow[t]{3}{*}{-0.10} & -0.1283705952 & 4.3699696768 & 3.8547397922 & $0.1845069812 \mathrm{E}+04$ \\
\hline & -0.3002436768 & 1.8399998613 & 2.1537487232 & $0.9359621384 \mathrm{E}+02$ \\
\hline & -0.5586547905 & 3.1719999323 & 3.0236122826 & $0.6148377803 E+03$ \\
\hline \multirow[t]{3}{*}{-0.14} & -0.5640610787 & 3.4599899025 & 3.2621355524 & $0.6184530063 \mathrm{E}+03$ \\
\hline & -0.5778812478 & 3.5639898774 & 3.3645241424 & $0.7068247924 \mathrm{E}+03$ \\
\hline & -0.5860065351 & 3.6279898115 & 3.4307327160 & $0.7692412993 \mathrm{E}+03$ \\
\hline \multicolumn{5}{|c|}{ Family IB } \\
\hline \multirow[t]{3}{*}{-0.10} & -0.1567788697 & 5.5999999777 & 4.5635886628 & $0.8575069327 \mathrm{E}+03$ \\
\hline & -0.2477538194 & 4.6099799796 & 2.9427998950 & $0.4121178481 E+02$ \\
\hline & -0.6582096977 & 5.4399605561 & 5.1051625828 & $0.7380508650 \mathrm{E}+04$ \\
\hline \multirow[t]{3}{*}{-0.14} & -0.1971564848 & 6.3070099842 & 7.7580254898 & $0.3273693864 \mathrm{E}+06$ \\
\hline & -0.2311124969 & 5.5799999916 & 4.8516031038 & $0.9091394693 \mathrm{E}+03$ \\
\hline & -0.6419742949 & 5.5699899860 & 5.4699203170 & $0.904886644 \mathrm{E}+04$ \\
\hline \multicolumn{5}{|c|}{ Family $N$} \\
\hline \multirow[t]{3}{*}{-0.10} & -0.2022400208 & 7.9300000000 & 9.4185594722 & $0.7405422727 \mathrm{E}+00$ \\
\hline & -0.2876472656 & 6.4580499925 & 1.6241609532 & $0.4502890350 \mathrm{E}+00$ \\
\hline & -0.6050866943 & 5.5459799961 & 1.5735755727 & $0.6420809871 E+02$ \\
\hline \multicolumn{5}{|c|}{ Family $N B$} \\
\hline \multirow[t]{3}{*}{-0.10} & -0.2553706741 & 7.8199999978 & 0.7525862132 & $0.2054833834 \mathrm{E}+01$ \\
\hline & -0.4406224670 & 7.1040099861 & 1.2581831552 & $0.1174944268 \mathrm{E}+01$ \\
\hline & -0.6006942480 & 6.8399999840 & 2.1016845842 & $0.1468309630 \mathrm{E}+01$ \\
\hline \multirow[t]{3}{*}{-0.14} & -0.3177686157 & 7.0080000000 & 1.3404607522 & $0.1288587444 \mathrm{E}+01$ \\
\hline & -0.4527148193 & 6.8360099989 & 1.7340313366 & $0.1204659066 \mathrm{E}+01$ \\
\hline & -0.6254412842 & 6.7880299996 & 4.1409371452 & $0.2989379106 \mathrm{E}+01$ \\
\hline
\end{tabular}

gives $a=d$, hence the stability condition is simply $|a|<1$, and this is the criterium used for this paper.

\section{Periodic Orbits}

For the three selected values of $\epsilon$, we obtain several families of periodic orbits as can be seen in Figs. 3-5. The analysis here presented is restricted to the intervals $-2.6 \leq x_{0} \leq 2.5$ and $0 \leq C \leq 10$.

Because both $\epsilon=-0.1$ and $\epsilon=-0.14$ belong to the same region of the parametric bifurcation plane [7], the obtained families are quite similar. For negative values of $x_{0}$ there are two equilibria which trigger the presence of new families, such as the group $\mathrm{N}$; the same situation happens for the equilibrium at $x_{0}>0$, with the $\mathrm{P}$ group of families. However, for $\epsilon=-0.3$, these equilibria no longer exist; hence the corresponding families are not present. Families of groups $\mathrm{N}$ and $\mathrm{P}$ will be analyzed in more detail.

In this section we describe some representative orbits of the families. In general, for each value of the parameter $\epsilon$ we take three orbits for three different values of the Jacobian constant $C$. In Tables 1 and 2 we give the initial conditions for the considered orbits: the period $T$ and the stability index $|a|$; we plot them and describe their main features.

\section{A. Family F}

The family (see Fig. 6) consists of retrograde orbits and highly unstable orbits. Along the orbit, the particle initially traces a short
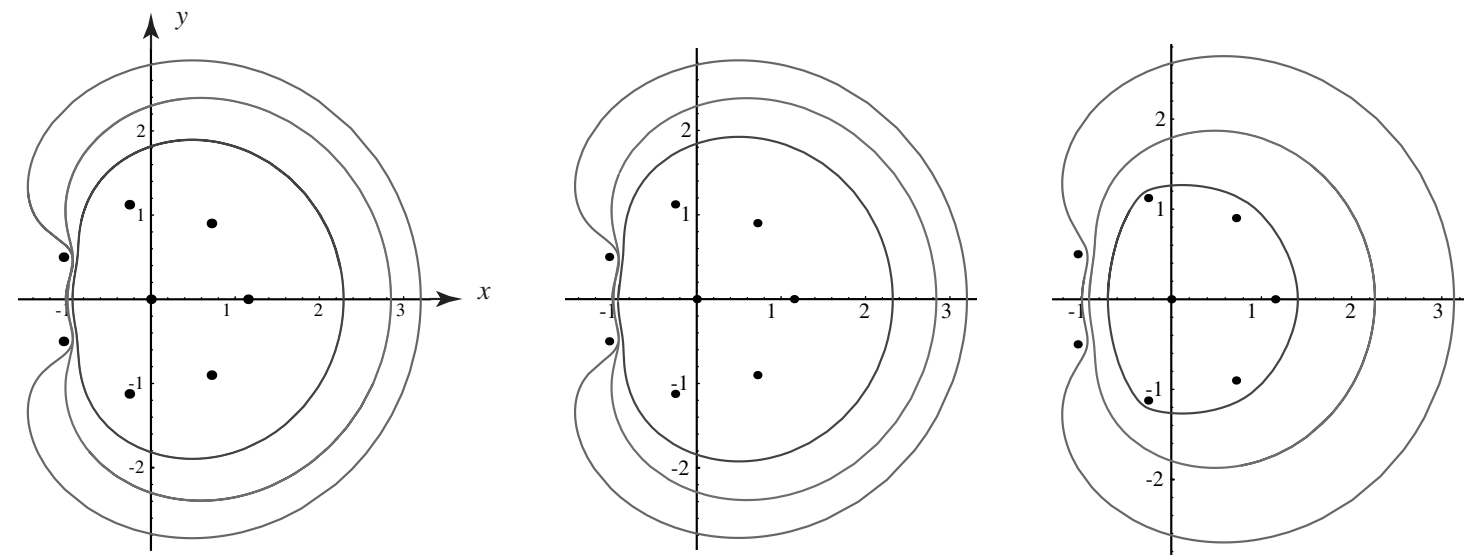

Fig. 6 Orbits of family $\mathrm{F}$ for $\epsilon=-0.1$ (left), $\epsilon=-0.14$ (center), and $\epsilon=-0.3$ (right) for different values of $C$. 

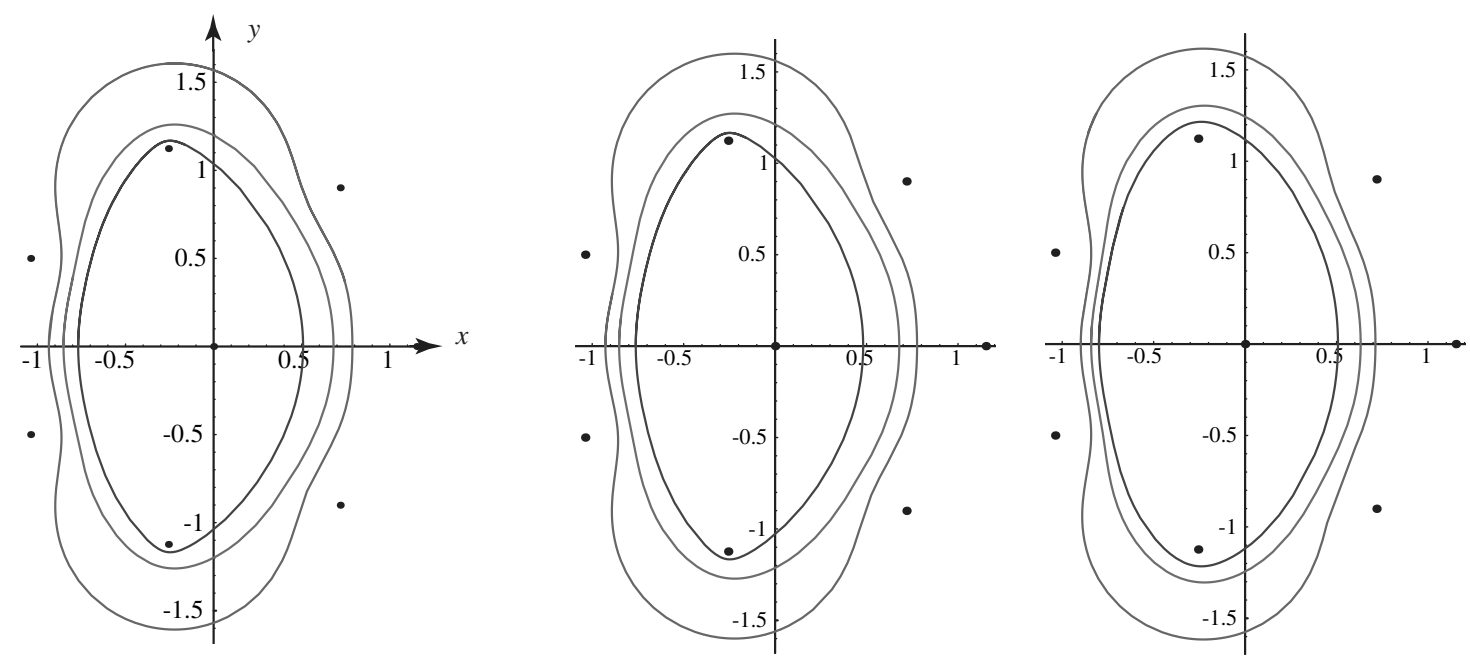

Fig. 7 Orbits of family $G$ for $\epsilon=-0.1,-0.14$, and -0.3 for different values of $C$.
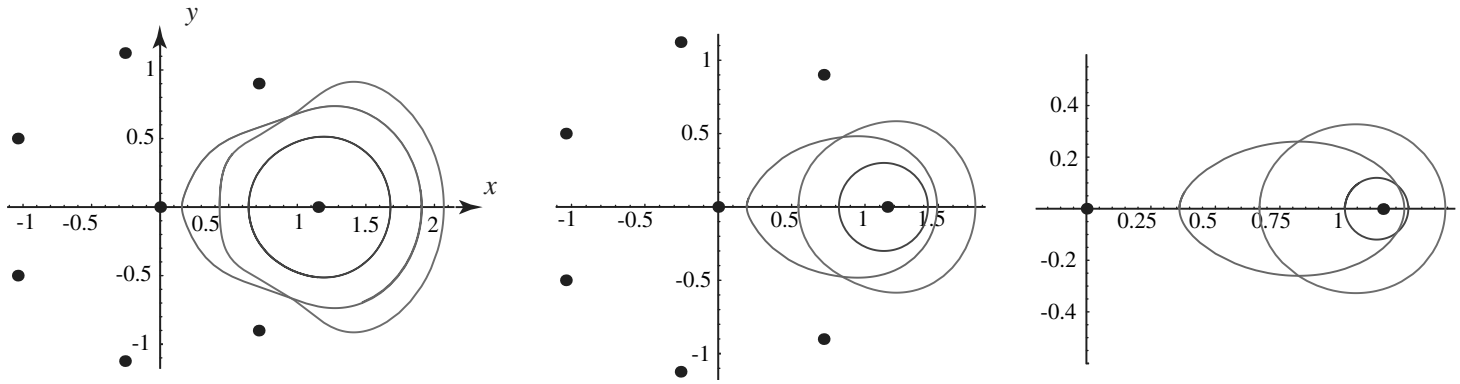

Fig. 8 Orbits of family $\mathrm{J}$ for $\epsilon=-0.1,-0.14$, and -0.3 for different values of $C$.
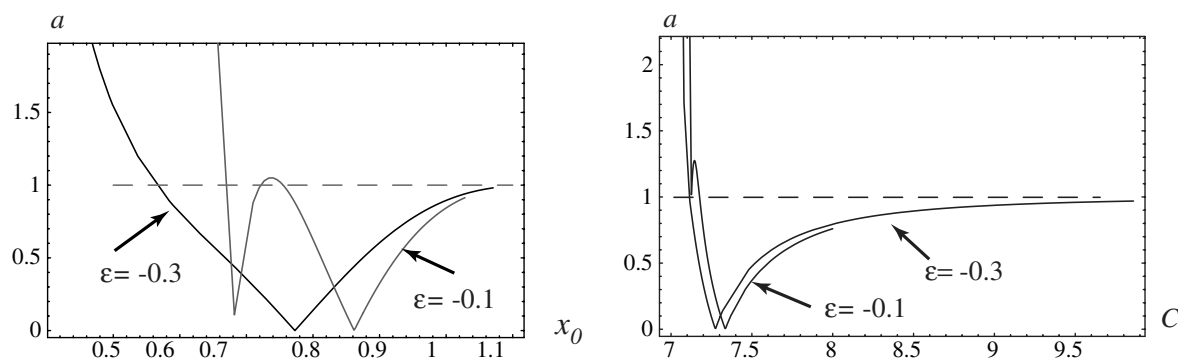

Fig. 9 Left: Stability index $a$ versus the initial condition $x_{0}$ for family J. Right: Stability index $a$ versus Jacobian constant $C$ for family K. The dashed line $a=1$ is the border of the stability regions.
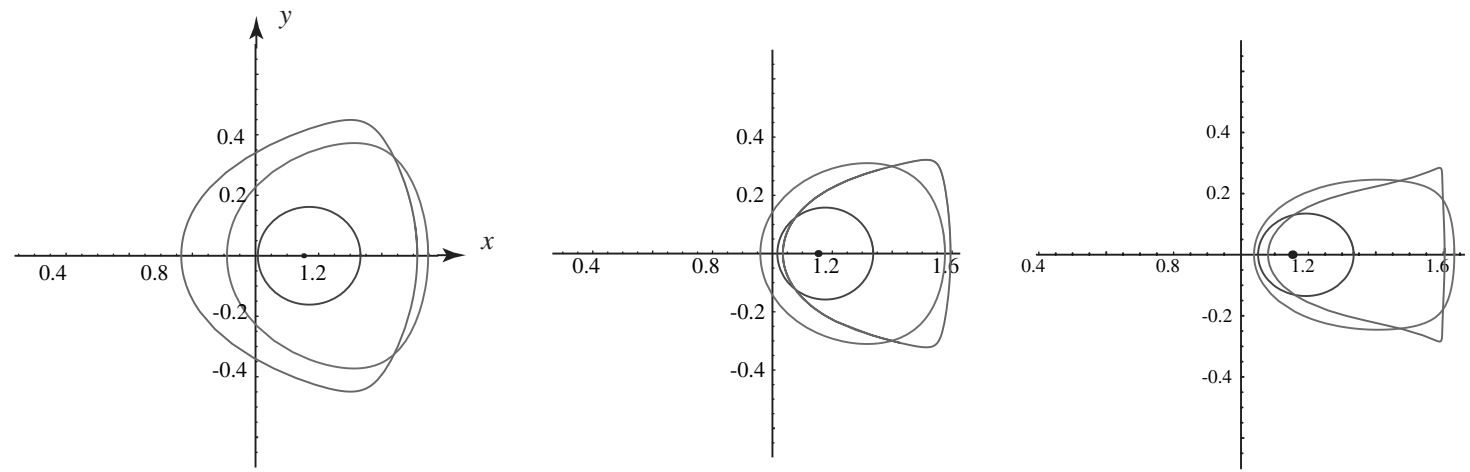

Fig. 10 Orbits of family $K$ for $\epsilon=-0.1, \epsilon=-0.14$, and $\epsilon=-0.3$. 

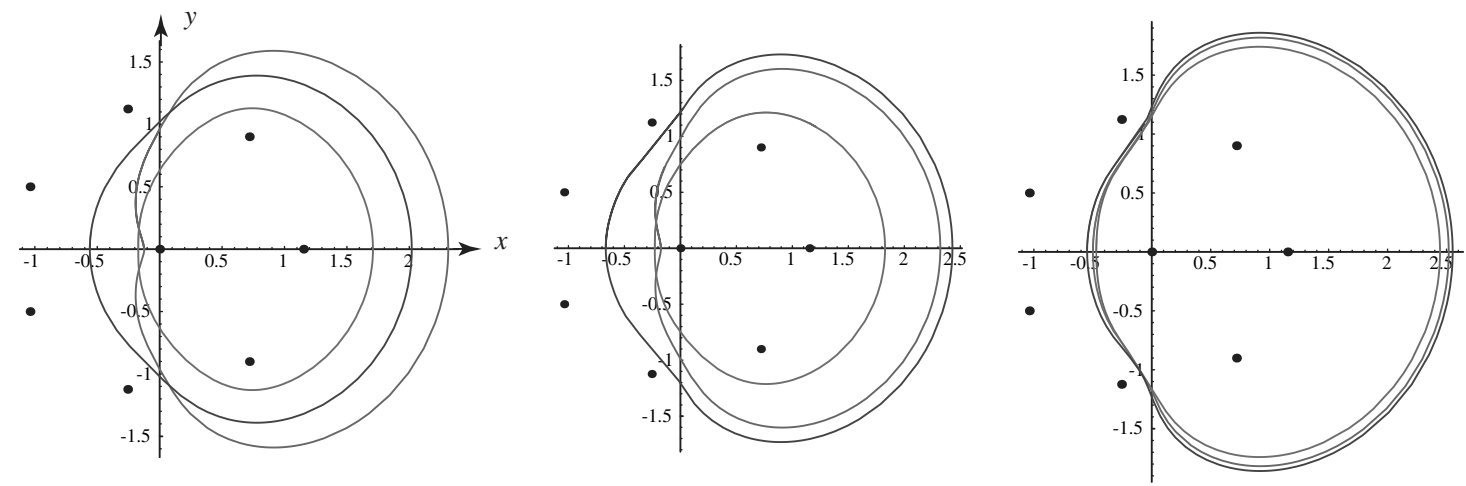

Fig. 11 Orbits of family I for $\epsilon=-0.1,-0.14$, and -0.3 for different values of $C$.
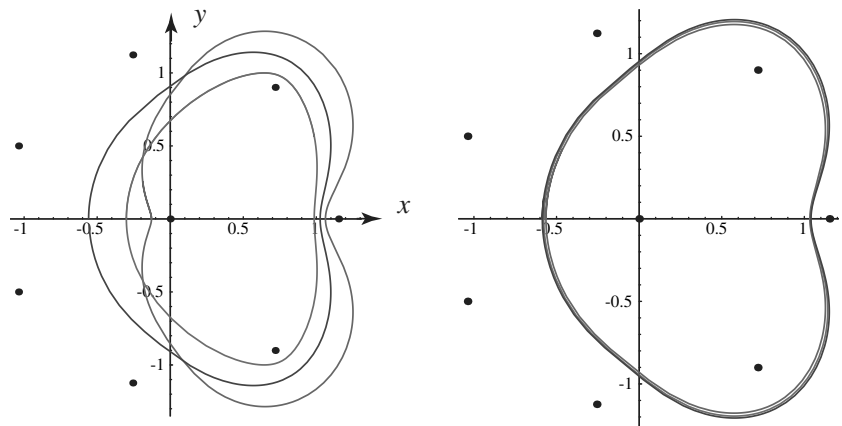

Fig. 12 Evolution of the orbits of family IA for $\epsilon=-0.1$ (left) and $\epsilon=-0.14$ (right) for different values of $C$.

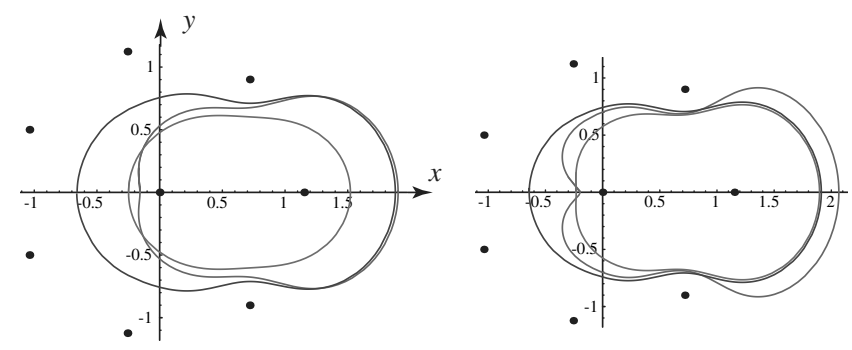

Fig. 13 Evolution of the orbits of family IB for $\epsilon=-0.1$ and $\epsilon=-0.14$ for different values of $C$.
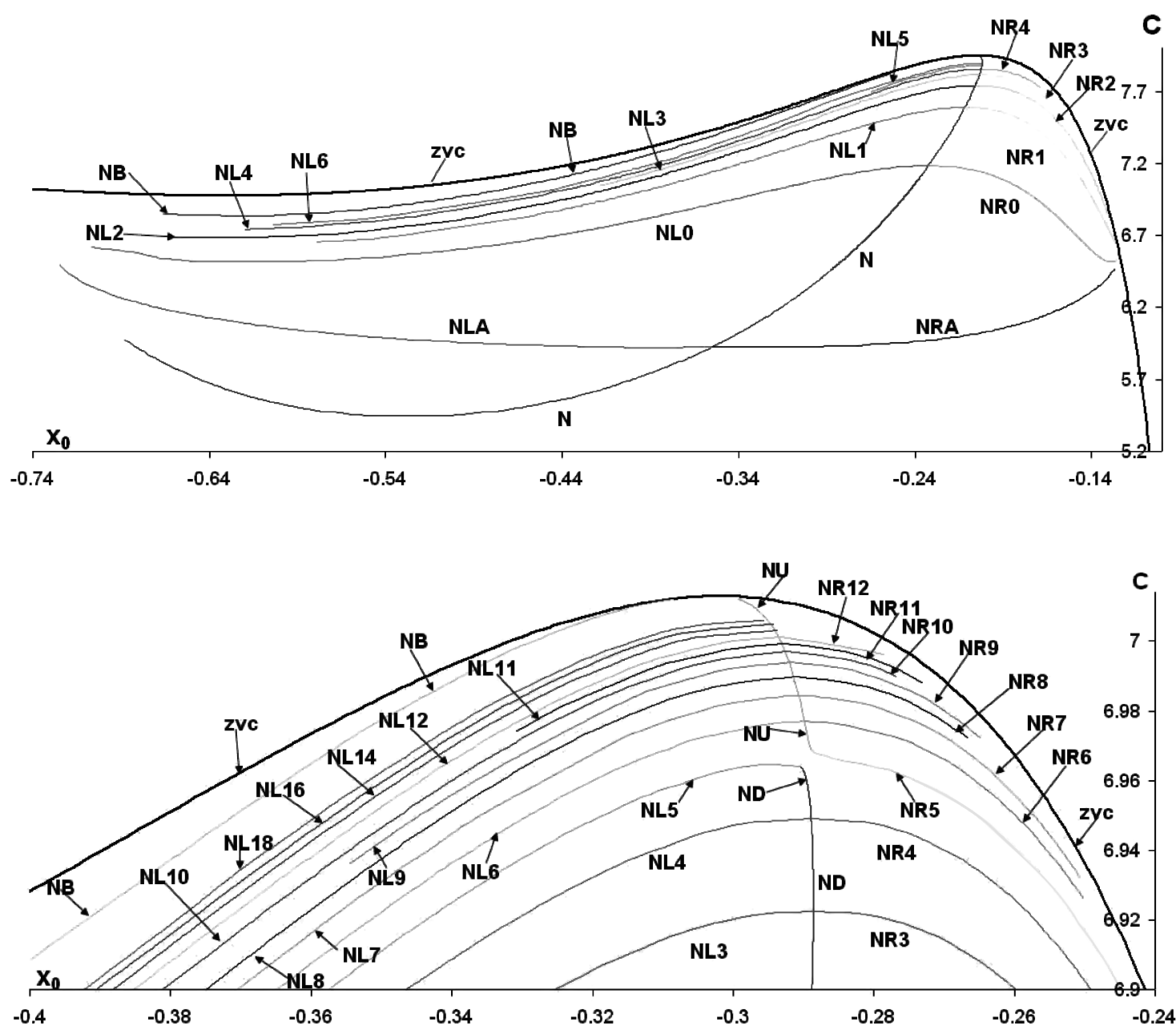

Fig. 14 The group $\mathrm{N}$ for $\epsilon=-0.1$ (top) and $\epsilon=-0.14$ (bottom). 

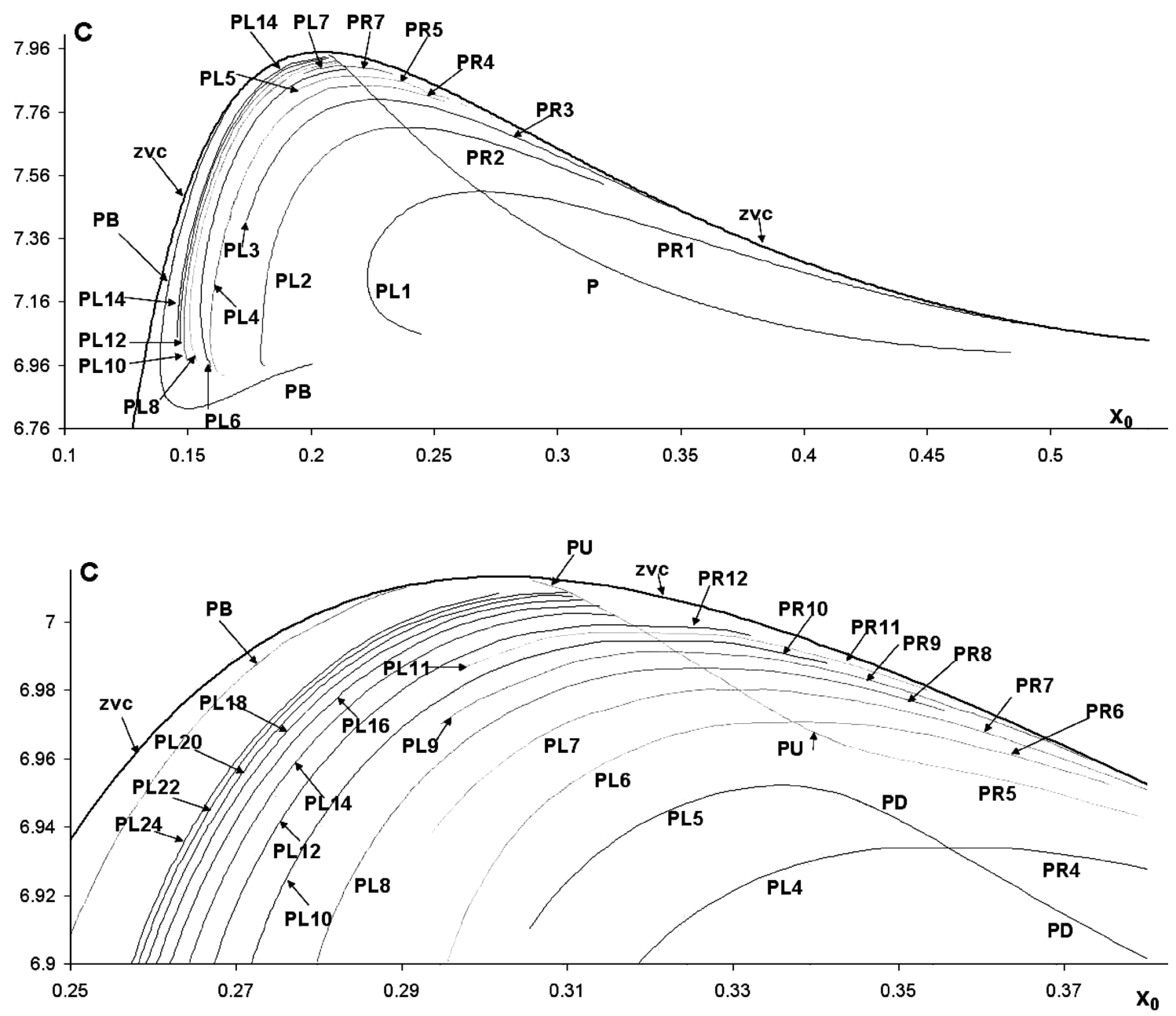

Fig. 15 The group $P$ for $\epsilon=-0.1$ (top) and $\epsilon=-0.14$ (bottom).

trajectory in the interior of the imaginary circle of the primaries, exits at a point between $P_{4}$ and $P_{3}$, and then describes a long arc outside the primaries and reenters the circle passing through the "entering" point which lies between $P_{6}$ and $P_{5}$, because of the symmetry. The sizes of the orbits, as well as the periods, decrease as the energy $C$ does.

\section{B. Family G}

Orbits of this family (Fig. 7) are retrograde and highly unstable. The particle initially traces a short trajectory in the interior of the imaginary circle of the primaries, and exits at a point between $P_{4}$ and $P_{3}$; then, it describes a short arc outside the primaries, reenters the circle at a point between $P_{3}$ and $P_{2}$, exits again between $P_{7}$ and $P_{6}$, and finally reenters passing through the entering point which lies between $P_{6}$ and $P_{5}$. The orbits size and their periods decrease with $C$, and the orbit tends toward peripherals $P_{3}$ and $P_{6}$.

\section{Family $\mathbf{J}$}

Orbits of this family only surround the primary $P_{1}$ (Fig. $\underline{8}$ ). On the plane $x_{0}-C$, the curve of this family is almost parallel to the zerovelocity curve (ZVC). Orbits are retrograde, and those orbits close to $P_{1}$ are almost circular. Although this problem is different from the restricted three-body problem (RTBP), we find some analogies between orbits of the two problems. Indeed, orbits of family $\mathrm{G}$ are analogous to the distant retrograde orbits (family $f$ ) of the RTBP.

Because the family $\mathbf{J}$ is not a one-to-one function with respect to the Jacobian constant $C$, the stability index $a$ is plotted in Fig. 9 (left) as a function of the initial condition $x_{0}$.

Table 3 Initial conditions for orbits belonging to families NL and PL and five or 10 peaks

\begin{tabular}{lcccc}
\hline \hline Peaks & $x_{0}$ & $C$ & $T$ & $a$ \\
Family $N L$ & & & & \\
5 & -0.2116460876 & 7.8099999993 & 3.5019735840 & $0.1000641341 \mathrm{E}+01$ \\
5 & -0.2972309165 & 7.5099899643 & 4.1253128768 & $0.1148160886 \mathrm{E}+01$ \\
5 & -0.4197301941 & 7.0439999195 & 5.8129305192 & $0.1113838421 \mathrm{E}+01$ \\
10 & -0.2096109772 & 7.9099999993 & 6.9178636126 & $0.1033634007 \mathrm{E}+01$ \\
10 & -0.3834640594 & 7.2319998773 & 10.5051656926 & $0.2644614031 \mathrm{E}+01$ \\
10 & -0.5994824720 & 6.7839997938 & 20.7402950044 & $0.3800075362 \mathrm{E}+00$ \\
Family PL & & & & \\
5 & 0.2044743881 & 7.7599899974 & 4.2427198744 & $0.1006608931 \mathrm{E}+01$ \\
5 & 0.1865643702 & 7.6299899917 & 4.6093293716 & $0.1028536039 \mathrm{E}+01$ \\
5 & 0.1738138351 & 7.4160099870 & 5.3994257274 & $0.1044997134 \mathrm{E}+01$ \\
10 & 0.1998510208 & 7.8999999982 & 7.5019098124 & $0.1095592914 \mathrm{E}+01$ \\
10 & 0.1713799706 & 7.7099899734 & 8.3529920478 & $0.2284642419 \mathrm{E}+01$ \\
10 & 0.1542268761 & 6.9659999220 & 17.0960685232 & $0.1510901099 \mathrm{E}+02$ \\
\hline \hline
\end{tabular}



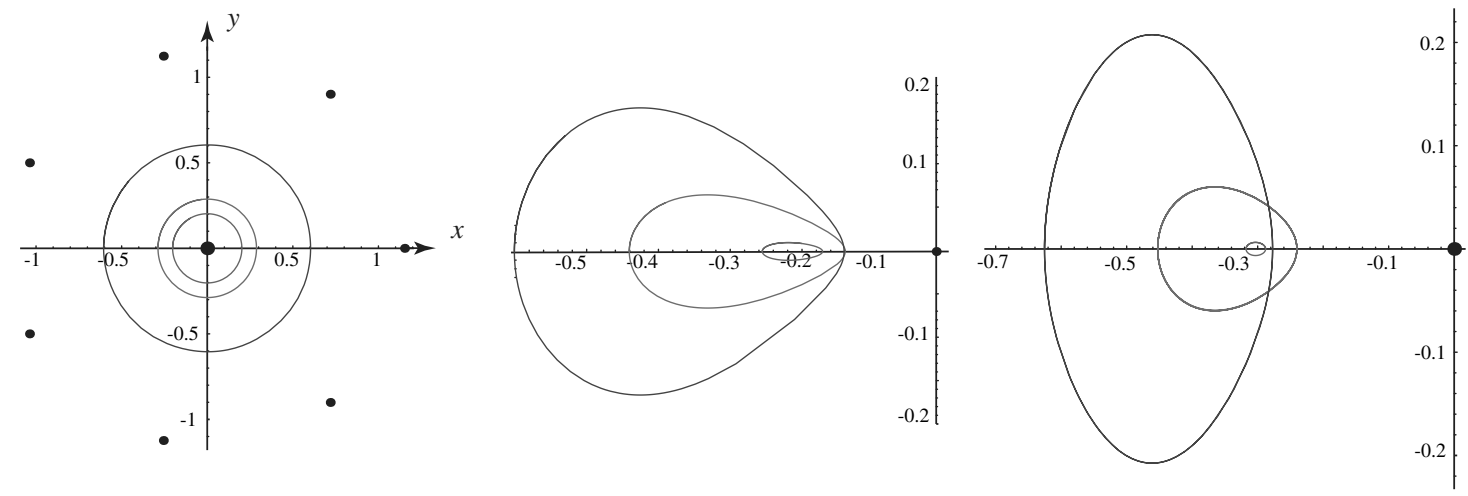

Fig. 16 Orbits of family N (left) and of family NB for $\epsilon=-0.1$ (center) and $\epsilon=-0.14$ (right).
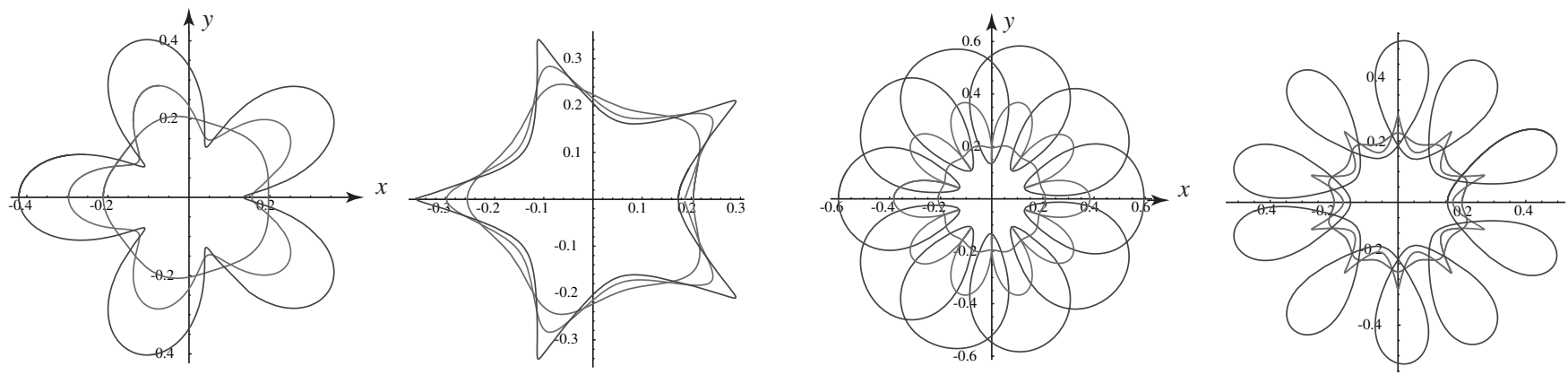

Fig. 17 Orbits of families NL and PL with five peaks (two on the left) and with 10 peaks (two on the right).

\section{Family K}

In Fig. 10 we plot some orbits of this family. As happened with family $\mathrm{J}$, orbits only encircle $P_{1}$, but in this case, the orbits are direct. They seem to spring out from the critical point that is outside of the ring and on the $O x$ axis. The curve of the family $\mathrm{K}$ on the $x_{0}-C$ plane is almost parallel to the zero-velocity curve. Orbits close to the primary are stable and almost circular. As $C$ decreases, orbits have a segment with very small curvature; these bell-shaped orbits are highly unstable (Fig. 9, right).

We note again that family $\mathrm{K}$ is quite similar to families $g$ (and/or $\left.g^{\prime}\right)$ in Hill's or RTBP model.

\section{E. Family I}

In Fig. 11 we plot several orbits of family I for the three cases of the parameter $\epsilon$. The global behavior is similar. The orbits are retrograde and unstable. Orbits encircle primaries $P_{0}, P_{1}, P_{2}$, and $P_{7}$, whereas the remaining primaries are outside the orbits. The size of the orbits reduces with decreasing $C$. Initial conditions for the orbits plotted in Fig. $\underline{11}$ are in Table $\underline{2}$.

\section{F. Family IA}

In Fig. 12 we show the evolution of the orbits of family IA for $\epsilon=-0.1,-0.14$, since this family does not exist for $\epsilon=-0.3$. On the plane $x_{0}-C$ (Figs. 3 and 4 ), the curves for families I and IA look very similar and are very close to one another. However, orbits of these two families are quite different indeed: in Family IA the peripheral $P_{1}$ is outside the orbits, as we can see in Fig. 12. The family is made of retrograde and highly unstable orbits. When $\epsilon$ decreases, the curve of the family on the $x_{0}-C$ plane appears at higher values of $C$.

\section{G. Family IB}

Orbits of this family encircle primaries $P_{0}$ and $P_{1}$ (see Fig. 13). The family is made of retrograde and unstable orbits.

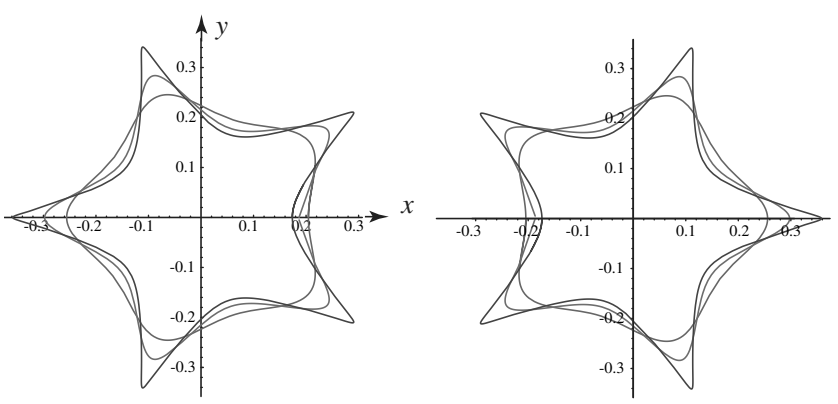

Fig. 18 Orbits of families PL (left) and PR (right). They are $y$ symmetric to each other.

\section{Groups of Families of N's and P's}

For negative values of $\epsilon$ there are two groups of families, namely, N's and P's (thus dubbed because they are obtained for negative and positive values of the initial condition $x_{0}$, respectively). Families $\mathrm{N}$ and $\mathrm{P}$ spring from the unstable collinear equilibria (see Figs. 3-5), and from these families emanate many new families denoted $\overline{\mathrm{P}} \overline{\mathrm{L}}$ 's, NL's (L for left) and PR's, NR's (R for right), as shown in Figs. 14 and 15 . The collinear equilibria are determined by the nonsphericity of the central primary, and they do not exit for $\epsilon$ smaller than a certain bifurcation value [7]; for instance, for $\epsilon=-0.3$ this equilibrium does not appear. Consequently, we will analyze the orbits for $\epsilon=-0.1$ and $\epsilon=-0.14$.

For a certain value of the parameter $\epsilon \in[-0.14,-0.1]$ the branch of family $\mathrm{N}$ is broken into two parts. The upper part is denoted as NU, whereas the lower part is denoted as ND. The breaking point coincides with the bifurcating point of the main family $\mathrm{N}$ from which the two families NL5 and NR5 emanate. Thereafter the lower part of family NU continues with the family NR5, while the upper part of family ND continues with NL5 (see Fig. 14). The same situation happens for the family $\mathrm{P}$, giving rise to the branches PU and PL (see Fig. 15). 

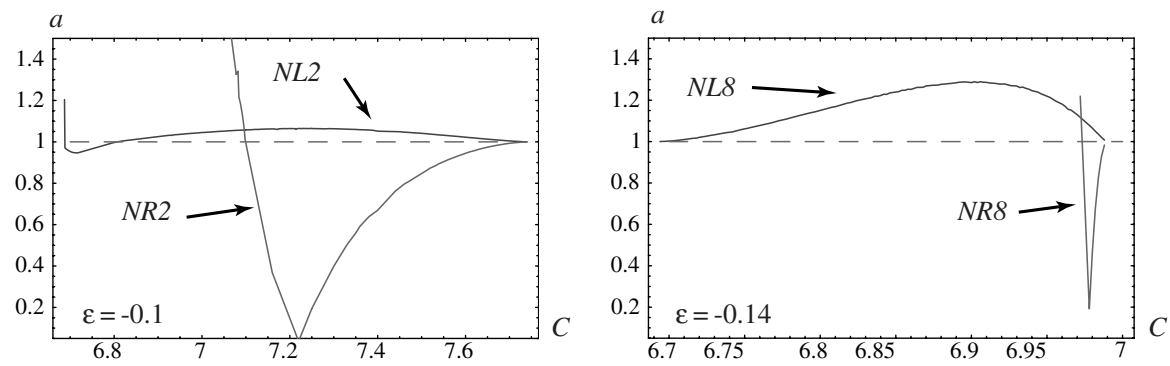

Fig. 19 Stability diagram for families NL and NR for two values of the parameter $\epsilon$. The points where NL and NR families coalesce at $a=1$ are bifurcation points belonging to the families $N$ (for $\epsilon=-0.1$ ) and $N U$ (for $\epsilon=-0.14$ ) (see Fig. 14).

\section{A. Families $\mathbf{N}$ and $\mathbf{P}$ (Main Families)}

Orbits belonging to these families are circular trajectories surrounding the central body. Orbits in $\mathrm{N}$ are retrograde, whereas orbits in $\mathrm{P}$ are direct. Some samples for $\epsilon=-0.1$ are given in Fig. 16 (left) and their initial conditions in Table 2.

The subfamily of $\mathrm{N}$ whose curve is very close to the zero-velocity curve (the boundary) is denoted by NB (see Fig. 14). These orbits do not surround any primary but the critical point, as shown in Fig. 16 (center and right). Orbits of $\mathrm{PB}$ are similar to the ones of $\mathrm{NB}$; however, the trajectories are direct.

\section{B. Families NL, NR, PL, and PR}

The curves of these families start from the $\mathrm{N}$ and $\mathrm{P}$ families (see Figs. 14 and 15).

In Fig. 17 there are orbits of branches NL and PL. We recall that orbits of families $\mathrm{N}$ and $\mathrm{P}$ are circular around the central body. As soon as we move along the branch NL (PL), there are undulations on the circle that soon after turn into a star-faced regular polygon. The number of vertices depends on how close the NL (PL) is to the equilibrium, in such a way that the more the family curve approaches the critical point the more vertices appear. For instance, the two orbits on the left in Fig. 17 have five peaks, whereas the two orbits on the right have 10 peaks. In the case of NL orbits, peaks point toward the central body, whereas in PL orbits, peaks point toward the exterior. This is the main difference between the two families. After a certain value of $C$, the peaks convert into loops giving rise to flower-shaped orbits. As is expected, the period increases with the size of the orbits. Initial conditions for orbits of these families are given in Table 3.

With respect to the branches bifurcated to the right of $\mathrm{N}$ and $\mathrm{P}$, that is, branches NR and PR, these orbits are almost identical to their corresponding ones in NL and PL families; the shape is the same but symmetrical to each other with respect to the $y$ axis (see Fig. 18). However, the stability is different as shown in Fig. 19.

\section{Conclusions}

The $N$-body ring problem is considered when the central mass is a prolate spheroid, which introduces a new parameter $\epsilon<0$. For this problem we consider several values of $\epsilon$ and find families of periodic orbits and their stability. The dynamics are very rich and the variety of orbits very wide; thus, we find orbits surrounding one, several, or no primaries, or even more complicated trajectories such as flowershaped periodic orbits.

The model considered in this paper is an extension of the Maxwell model for Saturn's rings and was proposed by the authors [7]. The paper may be considered as an extension of a previous work of Pinotsis [14], where the classical case $\epsilon=0$ was considered.

Several periodic orbits different from the ones considered here have been detected, as was expected when the grid-search method was used to find periodic orbits. The analysis of these orbits by a different method, such as the numerical continuation of families of periodic orbits, is in progress.

\section{Acknowledgment}

This work was supported by the Spanish Ministry of Science and Technology (Projects no. ESP2005-07107 and no. BFM200302137)

\section{References}

[1] Kalvouridis, T., "A Planar Case of the $n+1$ Body Problem: The "Ring" Problem," Astrophysics and Space Science, Vol. 260, No. 3 , 1998, pp. 309-325. doi:10.1023/A:1001739018698

[2] Scheeres, D. J., "On Symmetric Central Configurations with Application to Satellite Motion About Rings," Ph.D. Thesis, University of Michigan, Ann Arbor, MI, 1992.

[3] Maxwell, J. C., On the Stability of Motions of Saturn's Rings, Macmillan and Cia., Cambridge, 1859.

[4] Tisserand, F., Traité de Méchanique Céleste, Tome II, Gauthier-Vilars, Paris, 1889, Chap. 12.

[5] Kalvouridis, T., "Periodic Solutions in the Ring Problem," Astrophysics and Space Science, Vol. 266, No. 4, 1999, pp. 467-494. doi:10.1023/A:1002041820281

[6] Kalvouridis, T., "Zero Velocity Surface in the Three-Dimensional Ring Problem of $N+1$ Bodies," Celestial Mechanics and Dynamical Astronomy, Vol. 80, No. 2, 2001, pp. 133-144. doi:10.1023/A:1011919508410

[7] Arribas, M., and Elipe, A., "Bifurcations and Equilibria in the Extended $N$-Body Ring Problem," Mechanics Research Communications, Vol. 31, No. 1, 2004, pp. 1-8 doi:10.1016/S0093-6413(03)00086-7

[8] Elipe, A., On the Restricted Three-Body Problem with Generalized Forces, Astrophysics and Space Science, Vol. 188, No. 2, 1992, pp. 257-269. doi:10.1007/BF00644913

[9] Maneff, G., "La Gravitation et le Principe de l'Égalité de l'Action et de la Réaction," Comptes Rendus de l'Académie des Sciences, Serie IIa: Sciences de la Terre Planetes, Vol. 178, 1924, pp. 2159-2161.

[10] Arribas, M., Elipe, A., and Riaguas, A., "Non-Integrability of Anisotropic Quasi Homogeneous Hamiltonian Systems," Mechanics Research Communications, Vol. 30, No. 3, 2003, pp. 209-216. doi:10.1016/S0093-6413(03)00005-3

[11] Diacu, F. N., "Near-Collision Dynamics for Particle Systems with Quasihomogeneous Potentials," Journal of Differential Equations, Vol. 128, No. 1, 1996, pp. 58-77. doi:10.1006/jdeq.1996.0089

[12] Arribas, M., Elipe, A., Kalvouridis, T., and Palacios, M., "Homographic Solutions in the Planar $n+1$ Body Problem with Quasi-Homogeneous Potentials," Celestial Mechanics and Dynamical Astronomy, Vol. 99, No. 1, 2007, pp. 1-12. doi:10.1007/s10569-007-9083-8

[13] Arribas, M., Elipe, A., and Kalvouridis, T., "Central Configuration in the Planar $n+1$ Body Problem with Generalized Forces," Monografías de la Real Academia de Ciencias de Zaragoza, Vol. 28, 2006, pp. 1-8.

[14] Pinotsis, A. D., "Evolution and Stability of the Theoretically Predicted Families of Periodic Orbits in the $N$-Body Ring Problem," Astronomy and Astrophysics, Vol. 432, No. 2, 2005, pp. 713-729. doi:10.1051/0004-6361:20040262

[15] Hénon, M., "New Families of Periodic Orbits in Hill's Problem of Three Bodies," Celestial Mechanics and Dynamical Astronomy, Vol. 85, No. 3, 2003, pp. 223-246. doi:10.1023/A:1022518422926

[16] Hénon, M., "Exploration Numérique du Problème Restreint. II Masses Égales, Stabilité des Orbites Périodiques," Annales d'Astrophysique, Vol. 28, Feb. 1965, pp. 992-1007.

[17] Hénon, M., and Guyot, M., "Stability of Periodic Orbit in the Restricted Problem," Periodic Orbits, Stability and Resonances, edited by G. E. O. Giacaglia, Reidel, Dordrecht, Holland, 1970, pp. 349-374.

[18] Broucke, R., and Elipe, A., "The Dynamics of Orbits in a Potential Field of a Solid Circular Ring," Regular and Chaotic Dynamics, Vol. 10, No. 2, 2005, pp. 129-143. 